\title{
Lunar science: An overview
}

\author{
StuART ROSS TAYLOR \\ Department of Earth and Marine Sciences, Australian National University, Canberra \\ and \\ Lunar and Planetary Institute, Houston Texas, USA. \\ e-mail: Ross.Taylor@anu.edu.au
}

\begin{abstract}
Before spacecraft exploration, facts about the Moon were restricted to information about the lunar orbit, angular momentum and density. Speculations about composition and origin were unconstrained. Naked eye and telescope observations revealed two major terrains, the old heavily cratered highlands and the younger mostly circular, lightly cratered maria. The lunar highlands were thought to be composed of granite or covered with volcanic ash-flows. The maria were thought to be sediments, or were full of dust, and possibly only a few million years old. A few perceptive observers such as Ralph Baldwin (Baldwin 1949) concluded that the maria were filled with volcanic lavas, but the absence of terrestrial-type central volcanoes like Hawaii was a puzzle.

The large circular craters were particularly difficult to interpret. Some thought, even after the Apollo flights, that they were some analogue to terrestrial caldera (e.g., Green 1971), formed by explosive volcanic activity and that the central peaks were volcanoes. The fact that the craters were mostly circular was difficult to accommodate if they were due to meteorite impact, as meteorites would hit the Moon at all angles. The rilles were taken by many as definitive evidence that there was or had been, running water on the lunar surface. Others such as Carl Sagan thought that organic compounds were likely present (see Taylor 1975, p. 111, note 139).
\end{abstract}

\section{Introduction}

The intersecting lines on the lunar surface were thought, for example, by Gilbert Fielder (Fielder 1961) to reflect a 'lunar grid' due to tectonic stress patterns of internal origin. It was widely held that tektites came not only from the Moon (Chapman 1963) but specifically from the crater Tycho or from lunar volcanoes (O'Keefe 1970). Others such as Harold Urey (Urey 1952) maintained that the Moon was essentially a primitive undifferentiated object and would provide evidence about the earliest days of the solar system. All these ideas were published in the serious scientific literature.

In 1967, Lunar Orbiter IV returned a picture of Mare Orientale that immediately explained the origin of the many large circular structures on the Moon and enabled a correct interpretation to be made, for example, of Mare Imbrium.
Meanwhile Gene Shoemaker had proposed, in 1962, that geological maps of the Moon could be made, using the principle of superposition and these were compiled, especially by Don Wilhelms (1987).

The Apollo data from the first sample return in July 1969 immediately swept away the earlier speculation. The first rock samples returned were basalts that were readily examined by conventional petrography, mineralogy, geochemistry and geochronology. The maria were shown to be composed of basaltic lava flows, derived by partial melting from the mantle, with ages between 3 and 4 billion years. These iron-rich lavas were an order of magnitude more fluid than terrestrial lavas, accounting for the large smooth maria and the absence of central volcanic cones.

In contrast to the maria, the rocks from the highlands were all breccias that had been smashed up by meteorite impacts and reassembled many times.

Keywords. Origin of Moon; magma ocean; late heavy bombardment; chemical composition. 
But the lunar highlands were mainly composed of anorthosite, not granite, the highland plains were ejecta sheets from large basin impacts, not siliceous volcanics and the 'lunar grid' was an artefact caused by the patterns of the overlapping basin ejecta blankets. Tektites were not derived from the Moon but were formed as splashed glass from terrestrial impacts (e.g., Taylor 1973; Koeberl 1994). The postulated calderas were all due to meteoritic, asteroidal or cometary impact. The possible presence of ice at the lunar South Pole remains unconfirmed (Campbell et al 2003).

Based on the radiometric ages from the returned lunar sample, a dated stratigraphy was established. However, the complex impact history had not affected the chemistry and the highlands were shown to be mostly formed of a twocomponent mixture; anorthosite and material with a strongly enriched incompatible trace element signature, labeled KREEP (Taylor 1975, 1982; Basaltic Volcanism 1981; Heiken et al 1991).

The moment of inertia (I/Mr ${ }^{2}$ ) value of the Moon is $0.3931 \pm 0.0002$, that is low enough to require a small density increase in the deep interior, in addition to the low-density crust. Data from the Lunar Prospector magnetometer have refined the estimate to a core with radius $340 \mathrm{~km}$ but the evidence for a core remains enigmatic. The siderophile elements are depleted in the Moon in accordance with their metal-silicate distribution coefficients, consistent with their removal into a metallic core. Although this is evidence of metal segregation, perhaps it occurred in precursor bodies prior to the formation of the Moon. In the planetesimal hypothesis, the terrestrial planets gradually accreted from a swarm of smaller bodies, many of which had themselves melted and differentiated into iron cores and silicate mantles. In the current hypothesis for lunar origin, one such Mars-sized body that had formed from many precursors, impacted the Earth. The upshot is that the depletion of siderophile elements now observed in the Moon might have occurred in any or all of these earlier bodies. (Taylor and Norman 1990).

In addition, seismic evidence and lunar free oscillation studies are consistent with the presence of $1-2 \%$ of partial melt in the deep interior, which may indicate that the deep interior is composed of silicate, rather than possessing a metallic core.

As a result of the Apollo missions, major conclusions were reached about the evolution of the Moon (Taylor 1975, 1982). At least $50 \%$ of the Moon and probably the whole Moon was shown to have been melted, soon after accretion. The thick feldspathic highland crust had crystallised and floated on this 'magma ocean'. The interior had crystallised by about 4.4 billion years into a zoned sequence of cumulate minerals. KREEP was shown to be derived from the final few per cent of residual melt from this magma ocean that had concentrated elements unable to enter the dominant mineral phases (olivine, pyroxenes, plagioclase), thus accounting for the high near-surface concentrations of incompatible elements such as K, REE, P, U, Th, Zr, Hf, etc. (Taylor and Jakes 1974). The bulk Moon was bone-dry, depleted in volatile and siderophile elements and apparently enriched in refractory elements. No pre-Apollo theories of lunar origin survived. By 1984, a consensus was reached that the Moon originated as the result of the glancing collision between the Earth and a differentiated large impactor (about the size of Mars), whose silicate mantle spun out to form the Moon.

Since that time, the Clementine and Lunar Prospector missions have added materially to our knowledge and understanding of the Moon (Zuber et al 1997). New geophysical data are available on mascons. These indicate that the outer portions of the Moon have been cold enough and strong enough to support the mantle uplift and lunar topography (that has a range of $16 \mathrm{~km}$ ) for over 4 billion years.

The new missions have also firmly established the importance of the South Pole-Aitken basin. At $2500 \mathrm{~km}$ diameter, it is the largest recognized basin on the Moon, exceeded only by the problematical Procellarum basin that may underlie much of the near-side. Most of the upper crust is missing so that the South Pole-Aitken basin provides a window into the lower crust. The projected visit to this site may answer the question whether the lower crust differs from the upper crust or is similar.

The geochemical mapping of the lunar surface has enabled a division of the highland crust into three terranes: South Pole-Aitken, feldspathic highlands and Procellarum-KREEP (Jolliff et al 2000).

The feldspathic highlands terrane represents the battered remnants of the original crust of the Moon that formed by flotation of feldspar from the magma ocean. It is dominated by anorthosite and constitutes most of the far-side highlands.

The Procellarum-KREEP terrane covers much of the near-side and is delineated by the high concentrations of Th measured by the Prospector gamma-ray instrument. This records the signature of KREEP. The KREEP signature is missing both on the far-side highlands and in the deeply excavated South Pole-Aitken basin. This suggests that KREEP, that represents the residue of incompatible elements, was ponded under the nearside during the final crystallisation of the magma ocean. 
Work by Laskar and colleagues (1993) suggest that the Moon stabilises the obliquity of the Earth. They found that in the absence of the Moon, the Earth would undergo $20-30^{\circ}$ excursions in obliquity over time scales of a million years. Thus the large mass and close proximity of the Moon apparently stabilises the obliquity of the Earth. This has important implications for climate and habitability of this planet and the evolution of species.

Considerable progress has been made in the past decade on the earliest history of the solar nebula prior to lunar formation. Runaway accretion formed many small planetesimals with some approaching the size of Mars within timescales of $10^{5}$ years after $T_{0}$ (4567 m.y.). Melting and internal differentiation of planetesimals took place within a few million years after $T_{0}$. These planetesimals accreted into larger bodies so that impact events capable of supplying the angular momentum of the Earth-Moon system were probably common in the early solar system (Canup and Righter 2000; Taylor 2001).

The peculiarities of the chemistry and dynamics of the Moon can be ascribed in one way or another, to the extraordinary events surrounding a giant impact. The stochastic nature of the giant impact hypothesis, the original depletion of volatile elements in the nebula, the unknown nature of the impactor, from which most of the Moon comes in the single impact models, the great assemblage of precursor planetesimals and the loss of volatiles during the impact make the task of geochemists attempting to reconstruct that distant event, model-dependent at best.

The latest revision of the single impact hypothesis by Canup (2004) has reverted to earlier Cameron-Benz models. In these models, a body about the size of Mars made a glancing impact with the Earth at a stage when the Earth was nearly fully grown and both bodies had formed iron cores and silicate mantles. The rocky mantle of the impactor spun out into orbit around the Earth, while the coherent iron core remained intact and accreted to the Earth shortly following the collision. The Moon accreted from the rocky material in orbit, accounting for the low density of the Moon compared to the Earth.

In order to accommodate the angular momentum of both bodies, the large Moon-forming impact occurred at a stage when the Earth was nearly fully accreted, probably around 100 million years after $T_{0}$. Models of the giant impact derive most $(>85 \%)$ of the material from the rocky mantle of the impactor, not from the Earth. There are clear differences between the composition of the Moon and that of the terrestrial mantle in support of this concept. These include the high FeO content of the Moon (13\%) compared to $8 \%$ in the terrestrial mantle.

It is also worth recalling that most of the debris from the collision is dispersed so that the mass of the Moon is only about 5\% of the original mass of the impactor so perhaps the Moon is not a representative sample of the impactor mantle. Following the giant impact, the Moon accreted from the impact-generated disk of debris effectively instantly on geological time scales.

The question whether the lunar heavy bombardment around 3.8 to 4.0 b.y. represents a spike (or cataclysm) or the tail end of accretion remains unresolved, although possible collisional events in the asteroid belt may have resolved the difficult problem of the source of a spike of impacting projectiles several hundred million years after the formation of the Moon. Alternatively the late outward movement of Uranus and Neptune may have sent a shower of impactors sunwards from the Edgeworth-Kuiper Belt (Levison et al 2004).

Among the unresolved problems, the major unknown is the nature of the deep interior. Ideas about the depth of the magma ocean vary between half and whole-Moon melting and will depend on the deep internal structure. The central density may be too low for an iron core but perhaps consistent either with an FeS or a silicate core. A tiny core of $1-3 \%$ of lunar mass seems possible with a radius, from the Lunar Prospector magnetometer data, of $340 \mathrm{~km}$. However, it seems difficult to form a metallic core without involving whole Moon melting. Moreover the deep olivine and orthopyroxene cumulates from the magma ocean are likely to contain few heat sources, making it difficult to account for the possible presence of partial melt around $1150-1450 \mathrm{~km}$ that appears to be present from the Apollo seismic data. In an alternative model, the absence of a core could imply that only half the Moon was melted, leaving a primitive silicate interior. This would contain sufficient $\mathrm{K}, \mathrm{U}$ and Th to generate some partial melt $(1-2 \%$ is required), (Jolliff et al, in press). Data that would be of immediate interest would be various geophysical observations. These include heat flow, that could resolve some geochemical questions about bulk composition, and seismic data that could establish the nature of lunar deep structure and the presence or absence of a metallic core. These data could refine our ideas about initial thermal history and the initial depth of the magma ocean.

Another unresolved problem is the thickness of the highland crust. It has been traditionally set at $60 \mathrm{~km}$ (10\% of lunar volume), from the Nakamura interpretation of the seismic data in the 1970s. Recent re-evaluations by Kahn and Moesgaard (2001) and Wieczorek (pers. comm.) suggest an 
overall thickness of about $52 \mathrm{~km}$. The crustal thickness is critical for estimates of the bulk composition of the Moon. A $60 \mathrm{~km}$ thick crust implies an $\mathrm{Al}_{2} \mathrm{O}_{3}$ content of $6 \%$ and a corresponding enrichment over solar nebula values (from the CI meteorites) for the other refractory elements. However a crustal thickness of $52 \mathrm{~km}$ would obviate this problem to some degree and make for a less refractory rich Moon, easier to accommodate in geochemical models of the solar nebula.

Another series of problems deal with events in the solar system before formation of the Moon and the history of the precursor material that is critical to the interpretation of the W-Hf isotopic systematics.

Such questions include whether the Moon is a unique object or are there bodies (asteroids) with similar histories (Vesta)? What was the nature of the impactor? What was the thermal history of the precursor material? The major depletion of water and volatile elements in this model before planetary or lunar formation is demonstrated by the general depletion in the inner solar system of volatile elements such as potassium, relative to the abundance of refractory elements such as uranium for Venus, the Earth, Mars and many classes of meteorites. In this model, the loss of $\mathrm{Rb}$ and other volatiles mostly took place close to $T_{0}$ as shown by the low ${ }^{87} \mathrm{Sr} /{ }^{86} \mathrm{Sr}$ initial ratios. Thus the planetesimals that accreted to form the four inner planets were volatile depleted and dry. Ice was present far out in the asteroid belt, and its melting produced the aqueous alteration present in the CI chondrites.

So was the volatile element depletion in the Moon due to the collision, or was it inherited from the impactor and is a memory of events in the earliest nebula as suggested by the low value of LUNI or did both the events contribute (LUNI is the initial ${ }^{87} \mathrm{Sr} /{ }^{86} \mathrm{Sr}$ ratio of the Moon; it records depletion of volatile $\mathrm{Rb}$ relative to refractory $\mathrm{Sr}$ very close to $T_{0}$ rather than occurring during the formation of the Moon, an event that happened perhaps 50 or 100 million years later, see Nyquist 1977).

What about the possible enrichment in refractory elements compared to the CI solar nebula abundances? Elements with condensation temperatures $>1100 \mathrm{~K}$ are present in the Moon in the same relative proportions as in the CI carbonaceous chondrites, that are taken as representative of the composition of the primordial solar nebula. There is no sign of coupled $\mathrm{Eu}-\mathrm{Yb}$ depletion or enrichment that are shown by many of the REE patterns in the refractory inclusions (CAI) in primitive meteorites. This indicates that the lunar precursor material, unlike the CAI inclusions in the X-wind (Wood 2004), was not subjected to repeated processes involving evaporation or condensation much above $1100 \mathrm{~K}$. Potassium has also not undergone any isotopic fractionation so that the potassium now in the Moon, like that in all other measured inner solar system material, including the Earth, has not undergone Rayleigh fractionation (Humayun and Clayton 1995). However the general enrichment of the Moon in such refractory elements as $\mathrm{Ca}, \mathrm{Al}$, and the $\mathrm{REE}$ might be consistent with derivation from single stage condensation from a high temperature cloud resulting from the giant impact. In summary, there are four major unknowns at present that limit our understanding of the Moon. These are crust, core, composition and cataclysm.

Harold Urey thought of the Moon as a primitive object. However, the lunar composition has clearly been strongly fractionated from that of the primitive solar nebula. But the depletion of the volatile elements in the Moon may date back to the early volatile element depletion at $T_{0}$. Thus ironically, the Moon may still preserve some memory of the earliest nebula, as Harold Urey suggested, after the long journey of the precursor material from primitive dust grains to the curious composition of the Moon.

\section{Acknowledgements}

This research was conducted at the Lunar and Planetary Institute, which is operated by the USRA under contract CAN-NCC5-679 with NASA. This is LPI Contribution No. 1268.

\section{References}

Baldwin R B 1949 The Face of the Moon; (Chicago: Univ. Chicago Press) 212 pp.

Basaltic Volcanism Study Project (BSVP) 1981 Basaltic Volcanism on the Terrestrial Planets; (New York: Pergamon) p. 245.

Campbell B A et al 2003 Radar imaging of the lunar poles; Nature 426 137-138.

Canup R M 2004 Dynamics of lunar formation; Annu. Rev. Astron. Astrophys. 42 441-475.

Canup R M and Righter K 2000 Origin of the Earth and Moon; (Tuscon: Arizona Univ. Press) Arizona, 555 pp.

Chapman D R 1963 On the lunar origin of tektites; J. Geophys. Res. 68 4305-4358.

Green J 1971 Copernicus as a lunar caldera; J. Geophys. Res. 76 5719-5731.

Fielder G H 1961 Structure of the Moon's Surface; (Oxford: Pergamon Press) UK, p. 162.

Heiken G, Vaniman D and French B M 1991 The Lunar Sourcebook; (Cambridge: Cambridge Univ. Press) UK, $736 \mathrm{pp}$.

Humayun M and Clayton R 1995 Potassium isotope geochemistry: Genetic implications of volatile element depletion; Geochim. Cosmochim. Acta 59 2131-2148.

Jolliff B L et al 2000 Major lunar crustal terranes; J. Geophys. Res. 105 4197-4216.

Jolliff B L et al 2005 New Views of the Moon; Amer. Min. Soc. (in press). 
Khan A and Mosegaard K 2001 New information on the deep lunar interior from an inversion of lunar free oscillation periods; Geophys. Res. Lett. 28 1791-1794.

Koeberl C 1994 Tektite origin by hypervelocity asteroidal or cometary impact; target rocks, source craters and mechanisms; Geol. Soc. Am. Spec. Paper 293 133-151.

Laskar J, Joutel F and Robutel P 1993 Stabilization of the Earth's Obliquity by the Moon; Nature 361 615-617.

Levison $\mathrm{H}$ et al $2004 \mathrm{~A}$ fairy tale about the formation of Uranus and Neptune and the lunar late heavy bombardment; Astron. Soc. Pacific Conf. Ser. 324152.

Nyquist L E 1977 Lunar Rb-Sr chronology; Physics Chem. Earth 10103.

O'Keefe J A 1970 Tektite glass in Apollo 12 samples; Science 168 1209-1210.

Taylor S R 1973 Tektites: A post-Apollo view; Earth Sci. Rev. 9 101-123.

Taylor S R 1975 Lunar Science: A Post-Apollo View (Cambridge, UK: Pergamon) p. 372.
Taylor S R 1982 Planetary Science: A Lunar Perspective. Lunar and Planetary Institute, Houston, Texas, USA, $481 \mathrm{pp}$.

Taylor S R 2001 Solar System Evolution: A New Perspective (2nd edn) Cambridge Univ. Press.

Taylor S R and Jakes P 1974 The geochemical evolution of the Moon; Proc. Lunar Sci. Conf. 5 1287-1306.

Taylor S R and Norman M D 1990 Accretion of differentiated planetesimals to the Earth; In: Origin of the Earth (Oxford Univ. Press) pp. 29-43.

Urey H C 1952 The Planets; Yale Univ. Press.

Wilhelms D E 1987 The Geologic History of the Moon; U.S. Geol. Surv. Prof. Paper No. 1348. U.S. Geological Survey, Washington, D.C.

Zuber M et al 1997 Topography of the Moon from the Clementine LIDAR; J. Geophys. Res. 1021591.

Wood J A 2004 Formation of chondritic refractory inclusions: the astrophysical setting; Geochim. Cosmochim. Acta 68 4007-4021. 